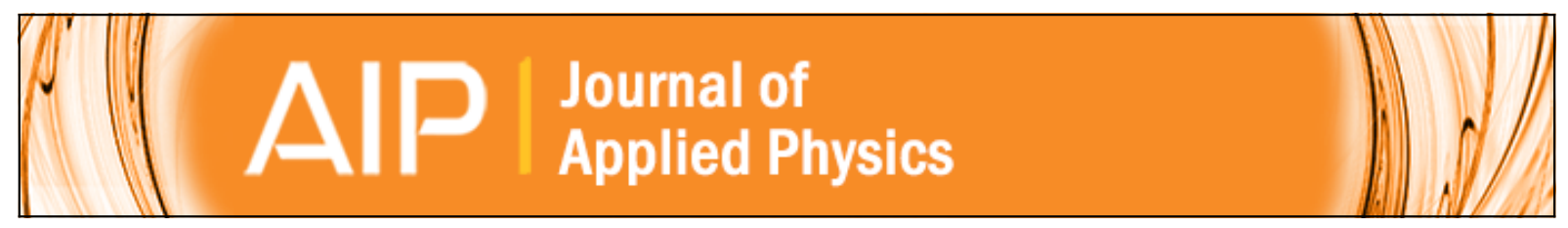

\title{
Unsteady nanoscale thermal transport across a solid-fluid interface
}

Ganesh Balasubramanian, Soumik Banerjee, and Ishwar K. Puri

Citation: Journal of Applied Physics 104, 064306 (2008); doi: 10.1063/1.2978245

View online: http://dx.doi.org/10.1063/1.2978245

View Table of Contents: http://scitation.aip.org/content/aip/journal/jap/104/6?ver=pdfcov

Published by the AIP Publishing

\section{Articles you may be interested in}

Heat conduction across a solid-solid interface: Understanding nanoscale interfacial effects on thermal resistance Appl. Phys. Lett. 99, 013116 (2011); 10.1063/1.3607477

Interfacial thermal transport between nanotubes

J. Appl. Phys. 106, 084302 (2009); 10.1063/1.3245388

Thermal transport through superlattice solid-solid interfaces

Appl. Phys. Lett. 95, 051907 (2009); 10.1063/1.3197012

Molecular dynamics simulations of thermal resistance at the liquid-solid interface

J. Chem. Phys. 129, 174701 (2008); 10.1063/1.3001926

Thermal transport across nanoscale solid-fluid interfaces

Appl. Phys. Lett. 92, 133105 (2008); 10.1063/1.2905281

MIT LINCOLN

LABORATORY

CAREERS

Discover the satisfaction of innovation and service

to the nation
- Space Control

- Air \& Missile Defense

- Communications Systems \& Cyber Security

- Intelligence, Surveillance and

Reconnaissance Systems
- Advanced

Electronics

- Tactical Systems

- Homeland

Protection

- Air Traffic Control

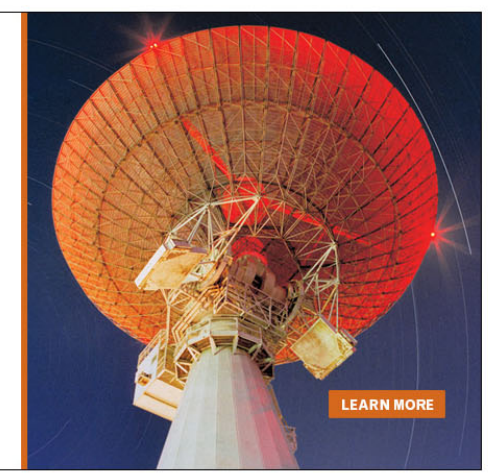




\title{
Unsteady nanoscale thermal transport across a solid-fluid interface
}

\author{
Ganesh Balasubramanian, Soumik Banerjee, and Ishwar K. Puri ${ }^{\text {a) }}$ \\ Department of Engineering Science and Mechanics, Virginia Polytechnic Institute and State University, \\ Blacksburg, Virginia 24061-0219, USA
}

(Received 12 April 2008; accepted 17 July 2008; published online 22 September 2008)

\begin{abstract}
We simulate unsteady nanoscale thermal transport at a solid-fluid interface by placing cooler liquid-vapor Ar mixtures adjacent to warmer Fe walls. The equilibration of the system towards a uniform overall temperature is investigated using nonequilibrium molecular dynamics simulations from which the heat flux is also determined explicitly. The Ar-Fe intermolecular interactions induce the migration of fluid atoms into quasicrystalline interfacial layers adjacent to the walls, creating vacancies at the migration sites. This induces temperature discontinuities between the solidlike interfaces and their neighboring fluid molecules. The interfacial temperature difference and thus the heat flux decrease as the system equilibrates over time. The averaged interfacial thermal resistance $R_{k, \text { av }}$ decreases as the imposed wall temperature $T_{w}$ is increased, as $R_{k, \text { av }} \propto T_{w}^{-4.8}$. The simulated temperature evolution deviates from an analytical continuum solution due to the overall system heterogeneity. (C) 2008 American Institute of Physics. [DOI: 10.1063/1.2978245]
\end{abstract}

\section{INTRODUCTION}

Since the length scales associated with nanostructures are comparable with those of the energy carriers that facilitate thermal transport, the corresponding nanoscale properties are different from those at the continuum. The role of the interfacial resistance ${ }^{1}$ during nanoscale thermal transport thus also differs from its characteristics at larger scales. ${ }^{2}$ Moreover, the structural details of a nanoscale interface have a significant influence on the local thermal properties. ${ }^{3,4}$ The attraction of liquid molecules to hydrophilic surfaces promotes structural ordering at interfaces, which enhances the local thermal conductivity. ${ }^{3,5}$ There is also evidence that the local intermolecular interactions can introduce temperature discontinuities across these interfaces. ${ }^{6}$ Kapitza $^{7}$ first discussed the existence of an interfacial contact resistance during heat transfer between liquid helium and heated metal surfaces such as copper and lead. The interfacial (Kapitza) resistance

$$
R_{k}=\Delta T / \dot{q},
$$

represents the ratio of the temperature drop at the interface to the normal heat flux across it. ${ }^{8,9}$ Thermal transport across nanoscale solid-liquid interfaces, e.g., those formed when nanoparticles and nanostructures are immersed in fluids, is also influenced by the locally large surface area to volume ratio. $^{10}$

\section{METHODOLOGY}

Atomistic simulations, such as those based on molecular dynamics (MD), which is a fundamental technique rooted in the principles of classical mechanics, ${ }^{11}$ can help explain interfacial effects ${ }^{12,13}$ during nanoscale thermal transport. ${ }^{14}$ Ex-

\footnotetext{
a) Author to whom correspondence should be addressed. Present address: Virginia Tech Department of Engineering Science and Mechanics, 223 Norris Hall, Blacksburg, VA 24061. Tel.: +1-540-231-3243. FAX: +1540-231-4574. Electronic mail: ikpuri@vt.edu.
}

amples of such MD studies ${ }^{15}$ include investigations of heat transfer between simple solid-liquid interfaces ${ }^{3,16}$ and of the bonding between liquid and solid atoms. ${ }^{17}$ These simulations have been limited to steady state investigations of nanoscale thermal transport across interfaces. ${ }^{4,6,16,18}$ Another limitation is that the heat flux is not typically determined in these simulations explicitly but rather a posteriori using the empirical Fourier heat conduction law. ${ }^{18}$ In order to address these two issues, we employ a nonequilibrium MD (NEMD) approach to investigate the transient thermal transport across a nanoscale interface in which the values of $\dot{q}$ are explicitly determined.

The interaction between a metal and an inert element is usually represented in MD simulations through a potential function, e.g., the Lennard-Jones (LJ) potential $u_{i j}$ $=4 \varepsilon_{i j}\left[\left(\sigma_{i j} / r_{i j}\right)^{1 / 12}-\left(\sigma_{i j} / r_{i j}\right)^{1 / 6}\right]$, where $r_{i j}$ denotes the scalar distance between sites $i$ and $j$, and $\sigma_{i j}$ and $\varepsilon_{i j}$ are the LJ interaction parameters. We model $\mathrm{Ar}-\mathrm{Ar}$ and $\mathrm{Fe}-\mathrm{Ar}$ interactions with LJ parameters, values of which are listed in Table I. ${ }^{19}$ MD simulations have also been able to reproduce the temperature discontinuities across solid-liquid interfaces by modeling the solid atoms with a harmonic potential function. ${ }^{18,20}$ While this is a better representation than the $\mathrm{LJ}$ model, which tethers solid atoms to their respective equilibrium positions, we employ the even more realistic embedded atom model (EAM), which incorporates many-atom interactions $^{21,22}$ that are otherwise neglected in a pairpotential scheme. ${ }^{23}$ For the EAM model, the total energy of a system of atoms $E=\Sigma_{i}\left[F_{i}\left(\bar{\rho}_{i}\right)+(1 / 2) \sum_{j(\neq i)} \Phi_{i j}\left(R_{i j}\right)\right]$, where the summation occurs over atoms $i$ and $j$. The embedding function $F_{i}$ denotes the energy required to embed an atom of

TABLE I. LJ interaction parameters for $\mathrm{Fe}$ and Ar.

\begin{tabular}{lcc}
\hline \hline Interaction parameters & $\varepsilon(\mathrm{eV})$ & $\sigma(\AA)$ \\
\hline $\mathrm{Ar}-\mathrm{Ar}$ & 0.0103 & 3.4 \\
$\mathrm{Ar}-\mathrm{Fe}$ & 0.0516 & 3.7 \\
\hline \hline
\end{tabular}




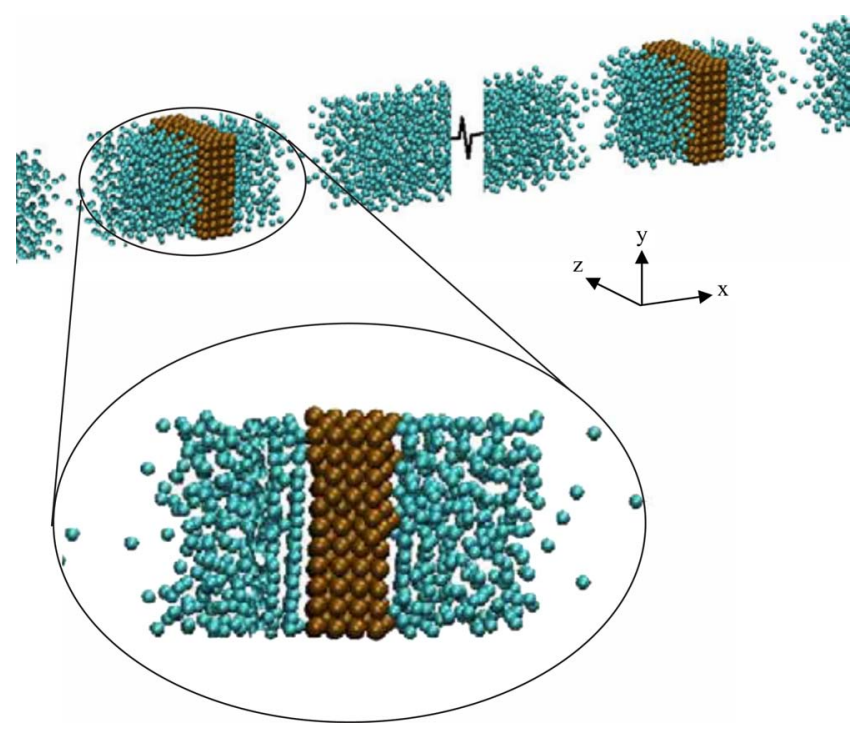

FIG. 1. (Color online) A three-dimensional view of the MD simulation domain in which Ar (cyan) fills a space of $28 \mathrm{~nm}$ between two $1 \mathrm{~nm}$ thick blocks of solid Fe. Fluid atoms on both sides of the walls indicate periodicity. A closeup orthographic view shows the observed Ar-atom layering at the solid-fluid interface.

type $i$ into the background electron density at the site $\rho_{i}$, and $\Phi_{i j}$ represents the pair-interaction between atoms $i$ and $j$ whose separation is $R_{i j}$. Here, the electron density of the solid is considered to be a linear superposition of the densities $\rho^{a}$ of the individual atoms. The relevant parameters for the EAM model are obtained from the literature. ${ }^{21}$

The simulation configuration is described in Fig. 1. The $3 \times 3 \times 58 \mathrm{~nm}^{3}$ cuboid contains two $3 \times 3 \times 1 \mathrm{~nm}^{3} \mathrm{Fe}$ blocks that restrain a liquid-vapor Ar mixture (with an initial $33 \%$ vapor volume fraction) between them. These solid Fe blocks extend from 14 to $15 \mathrm{~nm}$ and from 43 to $44 \mathrm{~nm}$ along the $x$-direction. The Ar liquid-vapor mixture consists of 3402 atoms that initially form a face centered cubic lattice, which equilibrates through the simulations. Periodic boundary conditions apply in all directions to eliminate wall effects so that the system is essentially infinite. The cutoff distance for the $\mathrm{LJ}$ interactions between both the metal-fluid and the fluidfluid atoms is $10 \AA$, or roughly 3 molecular diameters. Linear momentum is conserved for each simulation by subtracting the center of mass velocity of the group of solid atoms in each wall from each Fe atom within the group. Likewise, the center of mass velocity in each fluid reservoir is subtracted from each Ar atom in that reservoir. The number of atoms, total volume, and temperature of the solid walls in the closed system are held constant during the entire simulation.

The system is initialized at a temperature of $100 \mathrm{~K}$. During the first $300 \mathrm{ps}$, a velocity-rescaling temperature control is applied for all atoms. This ensures an initial equilibrium state for which the entire system is at a uniform temperature. Subsequently, for the next 700 ps, the temperature of the solid atoms is controlled while fluid atoms are allowed to behave freely and equilibrate. At 1000 ps, the Fe blocks are provided with a step increase to a higher temperature, which is thereafter maintained constant for the remainder of the simulation. The temperature of the Fe atoms is maintained by rescaling their velocities (i.e., their translational movement)

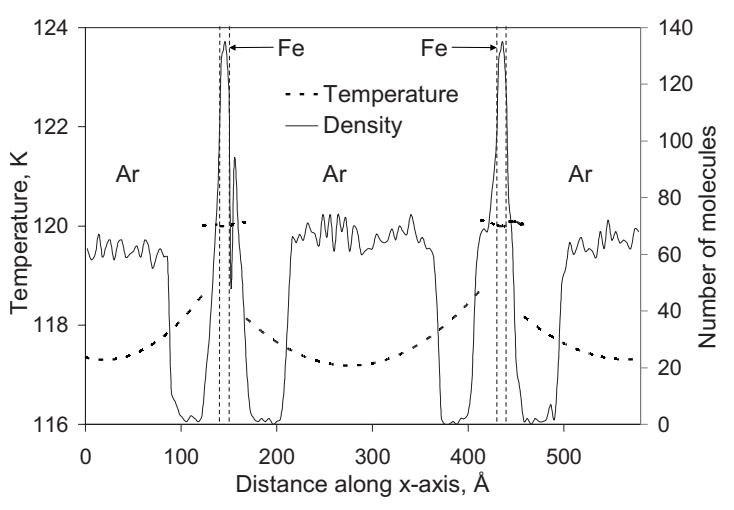

FIG. 2. The temperature (dotted line) and density (solid line) distributions across the $x$-direction after the system nears its steady state. The solid Fe walls are located between $14-15 \mathrm{~nm}$ and $43-44 \mathrm{~nm}$.

but keeping the overall kinetic energy constant. The instantaneous temperature $T=\left[2 /\left(n N k_{B}\right)\right] \sum_{i=1}^{N}(1 / 2) m v_{i}^{2}$, where $n$ denotes the molecular degrees of freedom $(=3$ for a monoatomic molecule), $k_{B}$ is the Boltzmann constant, $N$ is the number of atoms, $m$ is the atomic mass, and $v_{i}$ is the instantaneous velocity of an atom. The specified wall temperature $T_{w, s}$ is maintained by rescaling the velocity of each atom according to the relation $v_{i}^{\prime}=\left(T_{w, s} / T\right)^{1 / 2} v_{i}$. Thereafter, the simulation is continued with the modified velocities. Our MD simulations are based on the massively parallel LAMMPS $\operatorname{code}^{24}$ with successive 0.1 fs time steps.

\section{RESULTS AND DISCUSSION}

The fluid domain is divided into several $4 \AA$ thick slabs along the $x$-direction for the purpose of spatial characterization, and the density and temperature distributions within them are sampled at uniform time intervals. Figure 2 presents the averaged number density distribution of the $\mathrm{Fe}$ and Ar atoms when both Fe blocks are maintained at $120 \mathrm{~K}$. The figure shows that the fluid atoms adjacent to the solid walls migrate closer toward them due to the $\mathrm{Fe}-\mathrm{Ar}$ intermolecular interactions to form discrete interfacial layers in agreement with previous investigations. ${ }^{3,15,17,18,20}$ The consequent higher Ar atom density at the interface causes a local increase in the interfacial pressure so that the packed fluid layers are quasi-solid-like. Since the fluid is initially a liquidvapor mixture, this inhomogeneous density distribution occurs due to phase segregation. Ar atoms that are further removed from the walls do not experience the wall-vapor intermolecular attraction and thus remain homogeneously distributed. In contrast, the migration of the fluid Ar atoms toward the walls to form the quasicrystalline layers from proximal vapor-containing regions that are a few molecular diameters removed from the interfaces creates local vacancies. Essentially, Ar atoms closest to the walls exhibit a denser and more packed quasicrystalline behavior, those further removed from the interfaces behave as fluid, with vacancies separating these two regions.

Figure 2 also presents the average temperature (based on a local equilibrium approximation) of each $4 \AA$ thick slab in the domain. The Ar atom layers adjacent to each wall have a temperature nearly equal to that of the solid. This is followed 


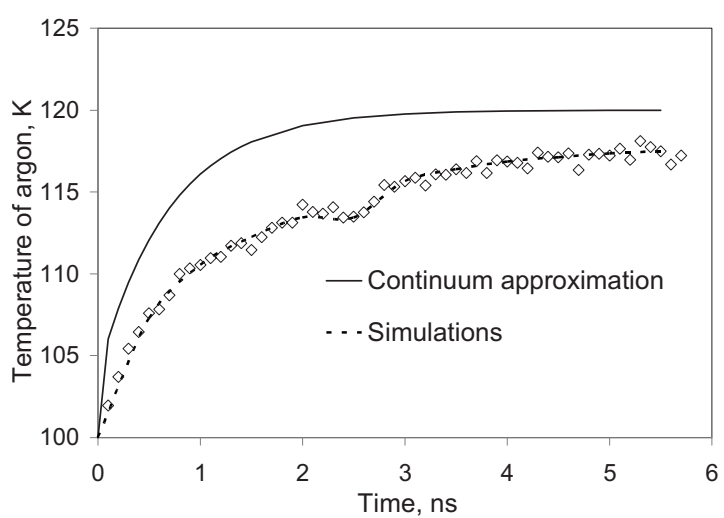

FIG. 3. The temporal evolution of the fluid temperature obtained explicitly from the simulations and empirically from an analytical solution to Fourier's law of heat conduction (assuming homogenous and constant properties).

by a sharp temperature drop in the fluid extending from approximately four molecular layers away from each wall. ${ }^{3,25}$ The Ar atoms in the four molecule thick interfacial regions show little tendency toward significant translational motion since, being attracted to the Fe atoms, they are held relatively immobile. These quasicrystalline Ar layers have a higher effective thermal conductivity than atoms in the corresponding fluid phase. ${ }^{25}$ In contrast to fluid behavior at the continuum scale, a significant temperature discontinuity occurs in the vacancies that follow the interfacial $\mathrm{Ar}$ atom layers since energy transfer through these sparsely populated regions is hindered. The thermal properties of nanoscale structures depend on the length scales of the energy carriers (for example, thermal phonons) and the system dimensions. Hence, if a domain is much larger than the mean free path of the energy carriers, only then is continuum theory expected to hold outside the interfacial region.

Figure 3 presents the temporal evolution of the characteristic fluid temperature, which is averaged over all slabs, as the simulation proceeds from its initial thermodynamic nonequilibrium state to a steady state. An analogous average temperature can also be obtained from the analytical solution of the continuum Fourier heat equation $\partial \theta / \partial \tau=\left(\partial^{2} \theta / \partial X^{2}\right)$, where $\theta$ denotes a dimensionless temperature $[T(x, t)$ $\left.-T_{w}\right] /\left(T_{i}-T_{w}\right), \tau$ is a dimensionless time $\alpha t / L^{2}$. Here, $\alpha$ $=1.12 \times 10^{-7} \mathrm{~m}^{2} / \mathrm{s}$ denotes the bulk Ar thermal diffusivity at the initial system temperature $T_{i}$ of $100 \mathrm{~K}, L$ is the length of the domain in the $x$-direction, and $X$ is a dimensionless length $x / L$. The boundary conditions correspond to fixed wall temperatures $T_{w}$. The solution follows the expression $\theta(X, \tau)=\sum_{n=1}^{\infty} 2 / n \pi[1-\cos (n \pi)] \sin (n \pi X) e^{-n^{2} \pi^{2} \tau}$.

The response of the MD simulations to thermodynamic disequilibrium differs from that of the analytical continuum solution. The simulation provides evidence of nonequilib-

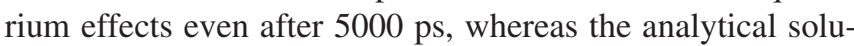
tion equilibrates by 3000 ps. Moreover, Fig. 3 also shows that the bulk temperature temporarily stabilizes after $\approx 2500 \mathrm{ps}$. We hypothesize that this occurs due to a temporary departure from the quasicrystalline interfacial layering of $\mathrm{Ar}$ atoms as the system equilibrates. This reordering impedes heat transfer for $\approx 200-400$ ps so that the fluid temperature remains virtually constant. Thermal transport re-

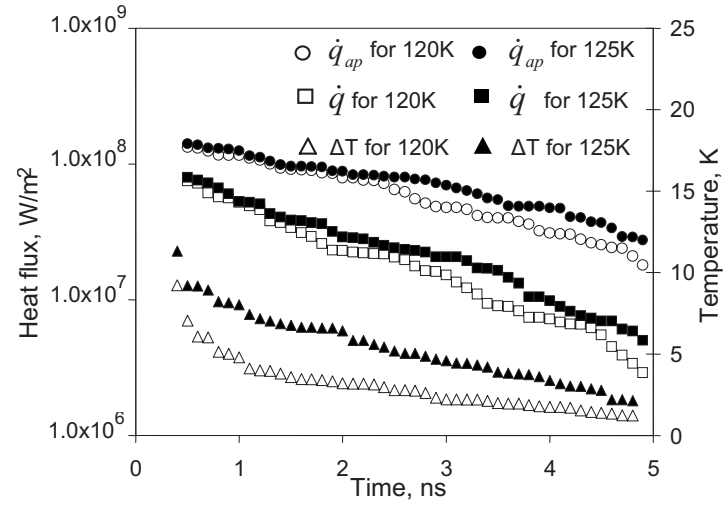

FIG. 4. The temporal evolution of the heat fluxes calculated using first principles and a quasicontinuum approach, and the interfacial temperature drop for two different wall temperatures of 120 and $125 \mathrm{~K}$.

sumes following the molecular rearrangement but at a slower rate. In contrast, the continuum analysis assumes homogenous system properties for all times, although the simulation results presented in Figs. 1 and 2 contain evidence to the contrary. We attribute the source for the discrepancy between the continuum analytical solution and the MD simulations to the heterogeneous system characteristics due to the interfacial layering of Ar atoms. This provides emphasis for our assertion that for values of the heat flux to be accurate, these must be determined explicitly from nanoscale simulations rather than implicitly using empirical continuum relations. If an appropriate system-specific correction factor for the bulk thermal conductivity is incorporated, the results from continuum theory can be expected to agree with the simulations.

We investigate this issue further. It is typical to determine the heat flux across nanoscale interfacial layers using a bulk thermal conductivity, i.e., by essentially making a quasicontinuum approximation. Instead we explicitly calculate the net energy flux $\dot{q}_{s l}$ from the solid molecules $s$ to the fluid molecules $l$, i.e., $\dot{q}_{s l}=\left(\dot{E}_{l s}-\dot{E}_{s l}\right) / 2$, where $\dot{E}_{l s}$ and $\dot{E}_{l s}$ denote the energy fluxes from the liquid to solid and solid to liquid phases, respectively. ${ }^{26}$ With this approach, the total thermal energy transferred from a high temperature wall to the low temperature fluid adjacent to it is $\dot{q}=\Sigma_{s} \Sigma_{l} \dot{q}_{s l}$, which also allows us to determine $R_{k}$ [Eq. (1)] explicitly from the simulation. Further, assuming that for a specified mass $m$ $=N_{\mathrm{Ar}} M_{\mathrm{Ar}}$ the approximate energy flux $\dot{q}_{\mathrm{ap}}=(m c / A)$ $\times\left(d T_{\mathrm{av}} / d t\right)$, where $N_{\mathrm{Ar}}$ and $M_{\mathrm{Ar}}$ denote the number of $\mathrm{Ar}$ atoms in the system and the molecular mass, $A$ is the interfacial area through which heat flows, $c$ is the constant volume specific heat of Ar, and $T_{\mathrm{av}}$ is the average fluid temperature, we obtain an expression for the approximate Kapitza resistance $R_{k, \text { ap }}=(A / m c) \Delta T /\left(d T_{\mathrm{av}} / d t\right)$.

The temporal variations in the heat fluxes, based on both the explicit and approximate approaches, and the temperature drops across the quasicrystalline Ar atom interface are presented in Fig. 4 for two initial wall temperatures of 120 and $125 \mathrm{~K}$. The behaviors of $\dot{q}, \dot{q}_{\text {ap }}$, and $\Delta T$ are similar for the two different initial wall temperatures. Since the temperature difference across the interface, which is the driving potential for heat transfer, diminishes over time, so does the heat flux from the high temperature solid to the equilibrating 


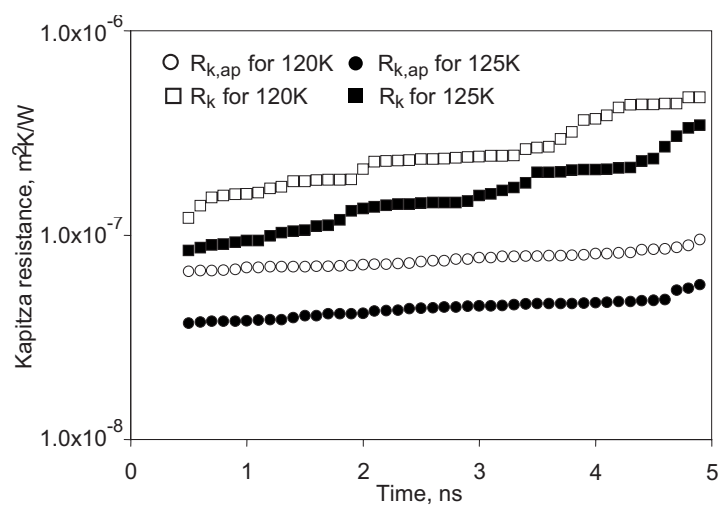

FIG. 5. The temporal evolution of the interfacial thermal resistances for two wall temperatures of 120 and $125 \mathrm{~K}$ (based on heat fluxes calculated using a first principles and a quasicontinuum approach). The interfacial temperature drop is the same for both methods at any instant and is calculated as a time average over $100 \mathrm{ps}$.

fluid for each case. Notably, there can be an almost one order of magnitude difference between the heat flux values predicted explicitly by the simulations and the approximate method.

Figure 5 presents the temporal variation in $R_{k}$ and $R_{k \text {,ap }}$ when the temporal values of $\Delta T$ are identical. Again, there is almost an order of magnitude difference in the values of these two interfacial resistances. Their behaviors are similar although their rates of increase are different. The interfacial thermal resistance increases over time since the decrease in the heat flux is greater than the decrease in the value of $\Delta T$.

We next examine the influence of wall temperature on the thermal resistance. For each simulated case, the value of $\Delta T$ is averaged over the period from 2000 to $4000 \mathrm{ps}$, and the heat flux $\dot{q}_{\mathrm{av}}=(1 / 2000) \sum_{i=2000 \mathrm{ps}}^{4000} \dot{q}_{i}$ to determine the average interfacial resistance $R_{k, \text { av }}$, which is presented in Fig. 6 with respect to increasing wall temperature $T_{w}$. As $T_{w}$ increases, the pressure within the fluid and hence on the quasicrystalline layers rises. Consequently, the interfacial molecular layers become more closely packed and the local

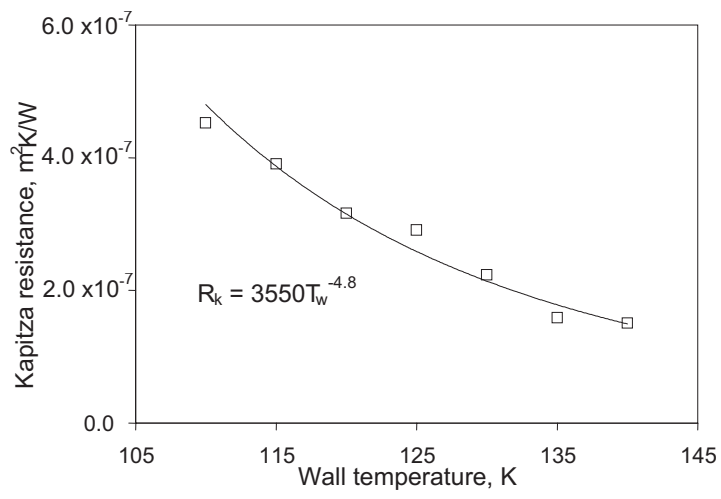

FIG. 6. Change in the Kapitza resistance (based on heat fluxes calculated using a first principles approach) with respect to varying wall temperature. The fluxes and temperature drops are averaged over 2000-4000 ps. intermolecular interactions are enhanced. Hence, thermal transport through these layers also increases, leading to a decrease in the interfacial thermal resistance according to the relation $R_{k, \text { av }} \propto T_{w}^{-4.8}$.

\section{CONCLUSION}

In summary, we corroborate that unsteady nanoscale thermal transport differs from that at the continuum scale. It takes longer for a nanoscale system to respond to an imposed temperature difference than predicted by an analytical continuum solution. The steady state temperature distribution between the quasicrystalline fluid interfacial layers adjacent to a solid wall and the rarified fluid-side vacancy-containing region following these layers exhibit a discontinuity due to phase segregation in the fluid. As the average temperature difference between the warmer interface and cooler fluid equilibrates over time, the heat flux also decreases. The resistance to heat transfer increases over time since the heat flux decreases more rapidly than does the average temperature difference. Overall, the interfacial thermal resistance decreases with increasing wall temperatures as $R_{k, \text { av }} \propto T_{w}^{-4.8}$.

\section{ACKNOWLEDGMENTS}

We thank the Virginia Tech Advanced Research Computing Facility for use of the terascale System X.

${ }^{1}$ E. T. Swartz and R. O. Pohl, Rev. Mod. Phys. 61, 605 (1989).

${ }^{2}$ D. G. Cahill, W. K. Ford, K. E. Goodson, G. D. Mahan, A. Majumdar, H.

J. Maris, R. Merlin, and S. R. Phillpot, J. Appl. Phys. 93, 793 (2003).

${ }^{3}$ S. Murad and I. K. Puri, Appl. Phys. Lett. 92, 133105 (2008).

${ }^{4}$ D. Chaudhuri, A. Chaudhuri, and S. Sengupta, J. Phys.: Condens. Matter 19, 152201 (2007).

${ }^{5}$ J. R. Henderson and F. Vanswol, Mol. Phys. 51, 991 (1984).

${ }^{6}$ R. Khare, P. Keblinski, and A. Yethiraj, Int. J. Heat Mass Transfer 49, 3401 (2006).

${ }^{7}$ P. L. Kapitza, J. Phys. (USSR) 4, 181 (1941).

${ }^{8}$ J. L. Barrat and F. Chiaruttini, Mol. Phys. 101, 1605 (2003).

${ }^{9}$ G. L. Pollack, Rev. Mod. Phys. 41, 48 (1969).

${ }^{10}$ J. A. Eastman, S. R. Phillpot, S. U. S. Choi, and P. Keblinski, Annu. Rev. Mater. Res. 34, 219 (2004).

${ }^{11}$ D. Poulikakos, S. Arcidiacono, and S. Maruyama, Microscale Thermophys. Eng. 7, 181 (2003).

${ }^{12}$ S. Murad and I. K. Puri, Nano Lett. 7, 707 (2007).

${ }^{13}$ S. Murad and I. K. Puri, Phys. Fluids 19, 128102 (2007).

${ }^{14}$ D. M. Heyes and N. H. March, Phys. Chem. Liq. 33, 65 (1996).

${ }^{15}$ L. Xue, P. Keblinski, S. R. Phillpot, S. U. S. Choi, and J. A. Eastman, J. Chem. Phys. 118, 337 (2003).

${ }^{16}$ C. S. Wang, J. S. Chen, J. Shiomi, and S. Maruyama, Int. J. Therm. Sci. 46, 1203 (2007).

${ }^{17}$ L. Xue, P. Keblinski, S. R. Phillpot, S. U. S. Choi, and J. A. Eastman, Int. J. Heat Mass Transfer 47, 4277 (2004).

${ }^{18}$ S. Maruyama and T. Kimura, Therm. Sci. Eng. 7, 63 (1999).

${ }^{19} \mathrm{~N}$. Lummen and T. Kraska, Nanotechnology 15, 525 (2004).

${ }^{20}$ T. Ohara and D. Suzuki, Microscale Thermophys. Eng. 4, 189 (2000).

${ }^{21}$ M. I. Mendelev, S. Han, D. J. Srolovitz, G. J. Ackland, D. Y. Sun, and M. Asta, Philos. Mag. 83, 3977 (2003).

${ }^{22}$ M. S. Daw, S. M. Foiles, and M. I. Baskes, Mater. Sci. Rep. 9, 251 (1993).

${ }^{23}$ R. A. Johnson and D. J. Oh, J. Mater. Res. 4, 1195 (1989).

${ }^{24}$ S. J. Plimpton, J. Comput. Phys. 117, 1 (1995); See http:// lammps.sandia.gov for more information.

${ }^{25}$ J. B. Freund, Phys. Fluids 17, 022104 (2005).

${ }^{26}$ T. Ohara, J. Chem. Phys. 111, 9667 (1999). 